\title{
Statistical Design of Experiments to Ensure "Rigor and Reproducibility" in Imaging Sciences
}

Vishwas N. Joshi ${ }^{1}$, Richard D. Powell ${ }^{1}$ and Eduardo Rosa-Molinar ${ }^{2}$.

1. Nanoprobes, Incorporated, 95 Horseblock Road, Unit 1, Yaphank, NY, USA.

2. University of Kansas, Department of Pharmacology and Toxicology and Neuroscience Graduate Program, Lawrence, Kansas, USA

In many scientific and engineering fields, experiments performed to increase knowledge and understanding are often conducted in a series of trials or tests that produce quantifiable, measurable outcomes.

The most common approach to experimental design employed by many scientists today is OneVariable-At-a-Time (OVAT), in which one variable at a time is changed, keeping all other variables in the experiment fixed (or constant); then, the change in the resulting outcome is observed. Quantifiable outcomes of the experiments are often measured using scientific instruments or equipment, e.g., in spectroscopic and microscopic techniques. The variations observed in the outcome of an experiment may stem from two sources: a) it may be a direct consequence of the intentional change in the input variable, usually the case if the outcome variable is correlated with the changing input variable; or b) it may be an error or variation in the measurement system (Fig. 1). Drawing correct logical conclusions when simultaneously working with nanoscale materials and devices and operating the measurement equipment near or at its performance limit, requires appropriate quantification of all sources of variability.

When several variables influence a particular output characteristic of a process or principle, the experiment must be designed to ensure that valid, reliable and sound conclusions can be drawn effectively, efficiently, and economically. Statistical Design of Experiments (SDoE) has been effective and efficient for general problem-solving, as well as for improving or optimizing product design and industrial manufacturing processes. SDoE is a methodology in which multiple variables can be changed and tested in parallel, and the impact of each variable can be resolved and quantified independently of other variables using quantitative statistical tools [1-3].

For example, in microscopy, the objective is to optimize a response or output variable. An example is the normalized intensity of a specific fluorescence signal (Fig. 2) from an immunofluorescence procedure that is influenced by several independent input variables, such as antibody concentration, number of reporter molecules per antibody, and acquisition time. Two steps are critical in SDoE : a) the definition of an approximation to a function or "model" to monitor the output, and b) the design of the experimental plan. First, different designs or models for fitting the response surface or the output can be tested by careful design of experiments (Fig. 2). Second, analysis of the response surface can be used for the approximation of both experimental and numerical responses. SDoE enables faster optimization while ensuring complete coverage of the response surface, which is much more laborious using OVAT.

Since brain research relies on imaging technologies, SDoE and its analytical tools can be used to address issues related to reproducibility in imaging sciences and to expedite imaging and analysis of biological systems at the lowest cost. In fact, SDoE could assist in meeting the challenges posed by the BRAIN 
(Brain Research through Advancing Innovative Neurotechnologies) initiative, which aims to produce a sophisticated understanding of the link between brain and behavior and to uncover new ways to treat, prevent and cure brain disorders [4-5].

\section{References:}

[1] M. H. Maathuis, Nat Methods 7 (2010), p. 247.

[2] D. Waltz and B. G. Buchanan, Science 324 (2009), p. 43.

[3] N. Blow, Nat Methods 6 (2009), p. 105.

[4] https://www.amstat.org/asa/files/pdfs/POL-StatisticsBRAIN_April2014.pdf.

[5] http://www.whitehouse.gov/share/brain-initiative.

\section{Conventional approach to optimization}

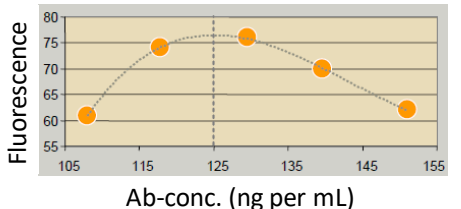

Ab-conc. (ng per $\mathrm{mL}$ )

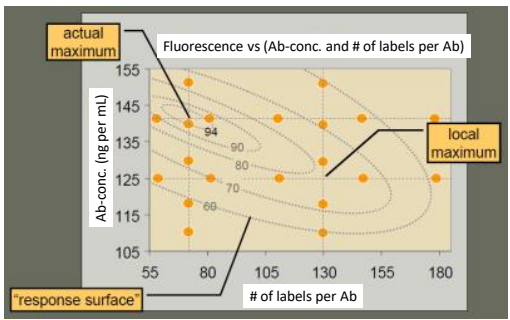

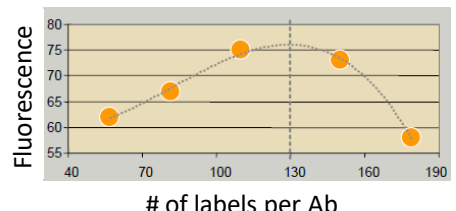

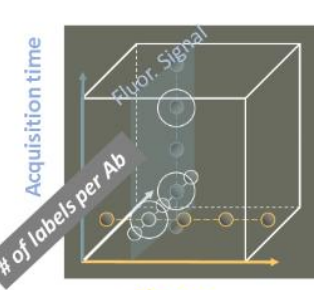

The problem: The optimum conditions obtained depend on the starting point; and inherent instrumental variations or errors.

Figure 1. Conventional approach to optimization of experimental conditions; change one parameter at a time and monitor its effect on the output.

\section{The Design of Experiments approach}

Rationally choose points throughout the cube to fully

represent the entire space.

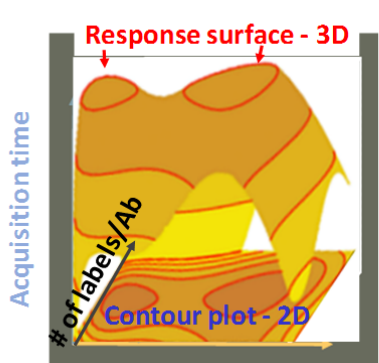

Ab-conc.
Outline

- Determining important reaction conditions

- Fractional factorial design

- Analysis of reaction condition effects

- Factorial design

- Estimation of the optimum conditions

- Response surface analysis

Response surface analysis can be used

for the approximation of both experimental and numerical responses

Figure 2. The Design of Experiments approach; rationally choose points throughout the cube to fully represent the entire space. 